\title{
Improvement of Sodium Status to Optimize the Efficacy of Renin-Angiotensin System Blockade
}

\author{
Gozewijn D. Laverman • Gerjan Navis
}

Published online: 4 October 2011

(C) The Author(s) 2011. This article is published with open access at Springerlink.com

\begin{abstract}
Blockade of the renin-angiotensin-aldosterone system (RAAS) offers superior renoprotection in the treatment of patients with hypertension, but the efficacy of RAAS inhibition strongly depends on sodium status, presumably in relation to extracellular volume status. Because assessing volume status by physical examination is challenging, 24-hour urine collection and NT-proBNP levels are useful tools for guiding volume management and achieving sodium status targets.
\end{abstract}

Keywords Hypertension - Chronic kidney disease ·

Blood pressure · Therapy · Renoprotection · Sodium ·

Renin-angiotensin system $\cdot$ Renin-angiotensin-aldosterone system $\cdot$ RAAS blockade $\cdot \mathrm{N}$-terminal pro-brain natriuretic peptide $\cdot$ NT-proBNP $\cdot 24$-hour urine collection .

Extracellular volume

\section{Introduction}

Treatment of hypertension is of paramount importance in chronic kidney disease (CKD). A series of intervention trials in the 1990s demonstrated the superiority of blockade of the renin-angiotensin-aldosterone system (RAAS) over other antihypertensives for renoprotection. This superiority

G. D. Laverman

Department of Internal Medicine, Division of Nephrology,

ZGT Hospital Almelo,

Almelo, The Netherlands

G. Navis $(\square)$

Department of Internal Medicine, Division of Nephrology, University Medical Center Groningen,

Hanzeplein 1,

9713 GZ, Groningen, The Netherlands

e-mail: g.j.navis@int.umcg.nl was even apparent when similar blood pressure was achieved, as first shown by the Ramipril Efficacy In Nephropathy (REIN) study, and is most likely due to specific renoprotective, antiproteinuric effects of RAAS blockade. Accordingly, RAAS blockade has become the therapy of choice for CKD, as recommended by the available guidelines.

Yet prescribing of these drugs, evidence-based as it may be, is not enough. The statistics on blood pressure control in patients with CKD are still poor, and the number of patients entering dialysis programs is constantly increasing. Tools to increase the efficacy of the prevention regimens based on RAAS inhibition are evidently necessary. It has become evident that for an optimal renoprotective effect of RAAS blockade, doses of these drugs must be high enough to reduce proteinuria, which may require higher doses than reduction of blood pressure alone. The hope that dual blockade with an angiotensin-converting enzyme (ACE) inhibitor and an angiotensin-receptor blocker (ARB) simultaneously might improve outcome in CKD has not been fulfilled, and there are data to suggest that dual blockade can even be harmful.

Interventions in pathways other than the RAAS are under investigation and may contribute to better renoprotection in the future. Here, we want to focus on a strategy to improve the efficacy of RAAS inhibitors that is being neglected in clinical practice: the targeting of sodium status.

\section{Sodium Status and RAAS Inhibition}

The efficacy of RAAS inhibition strongly depends on the actual sodium status of the patient. In fact, in circumstances of sodium retention and thus expanded extracellular volume, RAAS inhibiting drugs may be not effective at 
all. At the other end of the spectrum, blood pressure and renal function may drop dramatically in patients who are volume-depleted. It appears that this volume dependency is regarded as a disadvantage by many physicians. For example, ACE inhibitors with a short half-life, such as captopril, have been banned from some guidelines for treating hypertensive emergencies because their effect is considered too unpredictable, whereas in reality, the variability of the response is probably largely explained by the drug's dependency on volume status. The combination of RAAS inhibition with severe volume depletion can be dangerous, as typified by an 80 -year-old woman who enters the emergency ward with severe hypotension, acute renal failure, and hyperkalemia because she did not stop her ACE inhibitor when stricken with gastroenteritis. Does this problem imply that sodium status should somehow be taken into account or monitored in all patients for whom we prescribe RAAS blockade? How many doctors routinely consider the extracellular fluid status of their patients who are receiving an ACE inhibitor or ARB?

Whereas the issue of safety is obviously relevant, sodium status may be even more important from the point of view of achieving therapeutic efficacy. In our experience, obtained in patients with CKD as well as essential hypertension, targeting sodium status provides a tremendously powerful tool to increase the efficacy of RAAS blockade.

\section{Manipulating Sodium Status}

For this purpose, sodium status can be manipulated in clinical practice in well-established ways that include dietary sodium restriction, the use of diuretics, and the combination of these approaches. Actually, it should be unnecessary to mention sodium restriction in this respect, as it is included in all guidelines on treatment of hypertension and should be widely adopted - but it is not. This is a missed therapeutic opportunity, because even moderate sodium restriction (reducing the average 160 to $200 \mathrm{mmol}$ usually consumed by the general population and patients with CKD to about $100 \mathrm{mmol}$ per day) will reduce blood pressure by approximately $10 \mathrm{~mm} \mathrm{Hg}$ on top of the effect of RAAS blockade, and proteinuria will be reduced by some $30 \%$ ! A more or less similar effect can be obtained by adding a diuretic to the RAAS blockade. A thiazide diuretic is usually effective, or a loop diuretic may be used in patients with more advanced CKD, in which thiazide diuretics lose their efficacy. Of note, the effects of dietary sodium restriction and diuretic treatment are additive, enabling stepped volume titration. The aldosterone antagonists spironolactone and eplerenone offer an interesting alternative. While acting as a diuretic, these agents also may block the profibrotic effects of aldosterone at the organ level (vasculature, kidney, heart). Whether this effect translates into a better long-term outcome has not yet been proven, however.

The beneficial effects on blood pressure and proteinuria of targeting sodium excess during RAAS blockade have repeatedly been confirmed in short-term studies. Of note, recently presented post hoc data from randomized trials in nondiabetic and diabetic renal patients indicate that sodium excess is associated with worse long-term renal and cardiovascular outcome through the blunted efficacy of RAAS blockade. Although long-term prospective studies are still lacking, this finding supports the case for better management of sodium status and indicates that the benefit of even modest sodium restriction can be expected to be substantial, especially during RAAS blockade.

\section{Monitoring Volume Status}

To target sodium status, it is desirable that the intervention can be titrated; that is, that patients requiring additional correction of sodium excess can be identified and monitored. If blood pressure and proteinuria reach target values after the institution of sodium restriction and/or diuretic use, the intervention is clearly effective and no additional measures are required. All too often, however, clinical dilemmas arise when blood pressure control remains suboptimal, residual proteinuria remains, or both. Is volume correction still inadequate in this situation, and should it invariably be intensified? As a rule of thumb, residual ankle edema suggests persistent volume excess, but edema is not universally present in CKD patients (even those with proteinuria in the nephrotic range), and edema can occur in the absence of systemic volume overload.

Nevertheless, we are not completely empty-handed. Two simple tools can help to monitor the efficacy of volume targeting: 24-hour urine collection and the measurement of $\mathrm{N}$-terminal pro-brain natriuretic peptide (NT-proBNP). In our outpatient clinic, CKD patients collect 24-hour urines as part of their standard nephrologic care. These collections provide valuable information not only on diet (i.e., intake of sodium, potassium, and protein) but also on proteinuria and creatine clearance. Whereas 24-hour urine collection is often regarded with skepticism among clinicians because of its alleged inaccuracy, we take the point of view that the grim prognosis of CKD and the efforts required to implement a persistent change in dietary habits warrant proper instruction and training of the patient to this purpose, as an investment in future health. In our experience, patients are comfortable in doing these collections three times a year, as they gain useful feedback in return that helps them to make their efforts to reduce dietary 
sodium (and protein, if applicable) more successful. The 24-hour excretion of creatinine should be in line with body dimensions and estimated muscle mass and should be stable in consecutive collections, providing an adequate check for appropriate collection. However, one should be aware that even when sodium excretion (and hence, sodium intake) is acceptable, body sodium can be too high, as the state of sodium balance is governed not only by intake but also by the avidity of sodium-retaining mechanisms.

Therefore, we propose the use of NT-proBNP levels as an additional tool to guide volume management. NTproBNP is an indicator of the cardiac response to volume overload and has proven useful in patients with heart failure. We hypothesize that it also may be useful to guide volume management in CKD. We have found that NTproBNP levels are mildly increased in CKD patients without cardiac dysfunction, yet are well below the levels usually observed in heart failure. It is likely that the mildly increased NT-proBNP in CKD patients with proteinuria reflects their state of volume expansion. When we treated these patients with incremental volume intervention measures (sodium restriction and hydrochlorothiazide) added to RAAS blockade, the NT-proBNP levels were stepwise reduced towards normal. Interestingly, during each of the titration steps, an NT-proBNP level above normal predicted a good therapeutic response of blood pressure and proteinuria to the subsequent volume-intervention step. Once NT-proBNP was normalized, further volume depletion produced no added therapeutic benefit. We are currently investigating whether NT-proBNP can guide volume management in the clinical setting of renoprotection and could prevent potential adverse events of volume depletion, such as symptomatic hypotension, clinically relevant impairment of renal function, or gout. This tool may not only help to avoid acute adverse clinical effects, but also may help in titrating the patient to a volume status that affords optimization of the clinical efficacy of RAAS blockade, while avoiding possible long-term adverse effects of overzealous volume targeting, such as aggravation of chronic renal hypoxia leading to renal fibrosis, or the nongouty adverse effects of elevated uric acid on the cardiorenal risk profile.

\section{Conclusions}

The efficacy of RAAS inhibition strongly depends on sodium status, presumably in relation to extracellular volume status. Whether nonosmotic storage of sodium is also relevant in this respect remains to be investigated. As assessing volume status by physical examination remains a major challenge for any clinician treating patients with hypertension and kidney disease, NT-proBNP may prove to be a helpful tool in guiding volume management, and 24-hour urine collection adds valuable information on diet, proteinuria, and creatine clearance.

Disclosure No potential conflicts of interest relevant to this article were reported.

Open Access This article is distributed under the terms of the Creative Commons Attribution Noncommercial License which permits any noncommercial use, distribution, and reproduction in any medium, provided the original author(s) and source are credited. 